\title{
Mortalidad por defectos del tubo neural en México, 1980-1997
}

José A Ramírez-Espitia, + M en $C,{ }^{(1,2)}$ Fernando G Benavides, PhD, ${ }^{(2)}$ Marina Lacasaña-N avar ro, PhD, ${ }^{(1)}$ José M Martínez, Lic en Est, ${ }^{(2)}$ Ana M García, PhD, , (3) Joan Benach, PhD. ${ }^{(2)}$

\section{Ramírez-Espitia JA, Benavides FG, Lacasaña-Navarro M, Martínez JM, García AM, Benach J. Mortalidad por defectos del tubo neural en México, 1980-1997. \\ Salud Publica Mex 2003;45:356-364. El texto completo en inglés de este artículo está} disponible en: http://www.insp.mx/salud/index.html

\section{Resumen}

Objetivo. Describir la mortalidad en México por defectos del tubo neural, durante el periodo 1980-1997. Material y métodos. Las tasas anuales de mortalidad estatales y nacionales, por defectos del tubo neural, se calcularon por 10000 nacidos vivos. La tendencia temporal fue evaluada por el porcentaje de cambio anual obtenido mediante un modelo de regresión de Poisson. Se calculó la razón de mortalidad, to mando la media nacional como referencia. Las tasas y las razones se representaron gráficamente en mapas. Resultados. Durante el perio do la tasa bruta de mortalidad por defectos del tubo neural fue de 5.8 por 10000 nacidos vivos. La anencefalia fue el tipo de defecto más frecuente $(37.7 \%)$, seguida de la espina bífida sin hidrocefalia (31.6\%). La tendencia nacional de la mortalidad por defectos del tubo neural fue ascendente entre 1980 y 1990 (porcentaje de cambio anual 7.5 IC 95\% 6.5,8.6) y descendente entre 1990-1997 (porcentaje de cambio anual -2.3 IC 95\% $-3.6,-0.9)$. Conclusiones Las altas tasas de mortalidad por defectos del tubo neural fueron debidas principalmente a la elevada frecuencia de las anencefalias. El incremento observado parece no ser sólo atribuible a cuestiones puramente diagnósticas o de mejora en los registros. La influencia de factores asociados a estos defectos, como determinados

\author{
Ramírez-Espitia JA, Benavides FG, Lacasaña-Navarro M, \\ Martínez JM, García AM, Benach J. \\ Mortality due to neural tube defects \\ in Mexico, 1980-1997. \\ Salud Publica Mex 2003;45:356-364. \\ The English version of this paper \\ is available at: http://www.insp.mx/salud/index.html
}

Salud Pública de México lamenta el reciente deceso del maestro José Armando Ramírez Espitia, investigador del Instituto N acional de Salud Pública.

(1) Instituto Nacional de Salud Pública. Centro de Salud Ambiental. Instituto N acional de Salud Pública/O rganización Panamericana de la Salud. Cuernavaca, Morelos, México.

(2) Universidad Pompeu Fabra. Departamento de Ciencias Experimentales y de la Salud. Unidad de Investigación en Salud Laboral. Barcelona, España.

(3) Universidad de Valencia. D epartamento de Medicina Preventiva y Salud Pública, Bromatología, Toxicología y Medicina Legal.Valencia, España.

Fecha de recibido: 20 de junio de 2002 - Fecha de aprobado: 15 de abril de 2003

Solicitud de sobretiros: Dra Marina Lacasaña N avarro. Instituto N acional de Salud Pública, Centro de Investigación en Salud Poblacional,

Centro de Salud A mbiental IN SP/O PS. Avenida Universidad 655, 1er. Piso,

colonia Santa María A huacatitlán 62100 Cuernavaca, Morelos, México

Correo electrónico: mlacasan@ correo.insp.mx 
polimorfismos genéticos, la deficiencia de ácido fólico, la obesidad materna, la exposición laboral a plaguicidas y la pobreza deberán evaluarse mediante estudios específicos. El texto completo en inglés de este artículo está disponible en: http://www.insp.mx/salud/index.html

Palabras clave: mortalidad infantil; defectos del tubo neural; México be assessed in specific studies. The English version of this paper is available at: http://www.insp.mx/salud/index.html

Key words: infantile mortality; neural tube defects; Mexico
$\mathrm{L}$ a mortalidad infantil representa un problema de primera magnitud, especialmente en los países en desarrollo, y determina marcados contrastes entre aquellos pobres como Haití, cuya tasa de mortalidad infantil para el periodo 1990-1995 fue de 86 por 1000 nacidos vivos, y países ricos como Canadá, cuya tasa de mortalidad infantil para el mismo periodo fue de 7 por 1000 nacidos vivos. ${ }^{1}$ El riesgo de morir durante el primer año de vida es resultado de la interacción de diversos y complejos factores que se asocian con el estado de salud materno, el nivel de salud de una comunidad determinada, la calidad y acceso a los servicios de salud, las prácticas de salud pública, las exposiciones ambientales, los estilos de vida y las condiciones socioeconómicas, entre las que destaca la pobreza. ${ }^{1}$

Desde 1950, los países de América Latina y El Caribe han logrado disminuir en dos terceras partes su mortalidad infantil, pasando de una tasa de 127 defunciones por 1000 nacidos vivos en 1950, a una tasa de 40 muertes infantiles por 1000 nacidos vivos en $1995 .^{2}$ Entre las causas más frecuentemente asociadas con la mortalidad infantil en estos países destacan, en la actualidad, las enfermedades transmisibles y las afecciones del periodo perinatal. ${ }^{1}$ No obstante, la frecuencia de las enfermedades transmisibles ha ido disminuyendo mientras que las malformaciones congénitas (MC) han aumentado paulatinamente para consolidarse como la principal causa de muerte entre menores de un año de edad. ${ }^{3}$ El 89\% de las 589 mil muertes por MC reportadas en el mundo durante 1990 se suscitaron en la región de América Latina y El Caribe. ${ }^{4}$ En México, durante 1999, las MC ocuparon el décimo segundo lugar de la mortalidad general y el segundo de la mortalidad infantil, atribuyéndoseles $25.7 \%$ de las muertes en menores de un año de edad (37 defunciones por 10000 nacidos vivos). ${ }^{5}$ Si las estadísticas incluyeran todos los productos de la concepción la prevalencia de las MC sería aún mayor, ya que se considera que entre los abortos espontáneos y los abortos por prescripción médica, de los que hay un subregistro importante, entre 50 y $60 \%$ cursan con alguna anor- malidad cromosómica letal. ${ }^{6}$ Destaca, además, que en México las MC representan la principal causa de discapacidad entre quienes sobreviven con alguna de ellas. $^{7}$

Entre 40 y 70\% de las MC son de etiología desconocida. ${ }^{6,8,9}$ Los factores más comúnmente asociados con las MC son la edad de la madre al momento de la concepción, factores ambientales como las radiaciones ionizantes, el consumo de algunos fármacos específicos (antipsicóticos, antibióticos antihipertensivos), el consumo de alcohol y otras drogas, y la presencia de infecciones maternas (sífilis, rubéola, varicela, etcétera) o de patologías como la diabetes. ${ }^{6,8}$ Estos factores pueden ocasionar desde defectos del tubo neural (DTN) hasta ausencia total de extremidades, pasando por una gama de afecciones como lesiones encefálicas, hidrocefalia, lesión de los nervios craneales, labio y paladar hendidos, anomalías cardiacas, pulmonares, hepáticas y renales. También la carencia de algunos micronutrientes en la dieta materna, como el ácido fólico, se asocia con MC como DTN, malformaciones cardiovasculares, anomalías craneofaciales, etcétera. ${ }^{10-13}$ Asimismo, se ha señalado que ciertas exposiciones ambientales y laborales a diversas sustancias químicas, como los plaguicidas y algunos disolventes, pueden incrementar el riesgo de MC. ${ }^{14-17}$

Entre las MC, los DTN ocupan un capítulo especial por el daño implícito a diversas estructuras del sistema nervioso, que los hace comúnmente incompatibles con la vida. A principios de la década de 1980 en el ámbito mundial se estimó una prevalencia de 13.1 casos de DTN por 1000 abortos espontáneos, y de 10 casos de DTN por 10000 nacidos vivos. ${ }^{18}$ Según el Atlas Mundial de Defectos Congénitos, basado en algunos registros hospitalarios de MC, en 1998 México tuvo la prevalencia de anencefalia más alta del mundo: 15.8 por 10000 nacidos vivos. ${ }^{19}$ Durante el periodo 1994-1997 en México y en estados como Nuevo León, que cuenta con uno de los mejores índices de desarrollo del país, los defectos del tubo neural ocuparon el segundo lugar como causa de mortalidad infantil 
(21\%), precedidos por las malformaciones congénitas del sistema circulatorio $(42 \%){ }^{20}$

Los tipos de DTN más comunes son la anencefalia, la espina bífida y el encefalocele, que representan $95 \%$ de los casos. Estos padecimientos son resultado de un inadecuado proceso de cierre céfalo-caudal del tubo neural, que se suscita en el periodo de embriogénesis y que asegura el aislamiento del sistema nervioso central del exterior, pudiendo afectar la región cerebral, la medular o ambas e involucrando en mayor o menor grado tejidos meníngeo y óseo, músculos y piel., 21

En México, los DTN presentan una amplia variabilidad temporal y geográfica. No obstante, se ha señalado que 8 de cada 10 casos de DTN se concentran en estados de la zona centro del país, ${ }^{22}$ aunque hasta la fecha no se ha realizado ningún estudio que permita conocer con precisión la distribución temporal y espacial de estas patologías.

El objetivo de este trabajo es describir la mortalidad por DTN en México durante el periodo 1980-1997, tanto para el conjunto de la nación como para cada uno de los estados que la conforman.

\section{Material y métodos}

Las cifras anuales de población infantil del periodo 1980-1997 provienen de la base de datos estructurada por el Consejo Nacional de Población de México. Las cifras de mortalidad por DTN se obtuvieron de la base de datos de la Secretaría de Salud de México, cuya información proviene de los certificados de defunción codificados conforme a la Clasificación Internacional de Enfermedades (CIE 9a revisión). ${ }^{23}$ Las causas de muerte identificadas por DTN fueron la anencefalia (740.0), la espina bífida sin hidrocefalia (741.9), la espina bífida con hidrocefalia (741.0), el encefalocele (742.0), el craneorraquisquisis (740.1), y la iniencefalia (740.2). Esta base de datos incluye en un solo grupo a los menores de cinco años de edad. Debido a la imposibilidad de discriminar las muertes que correspondían específicamente a los menores de un año de edad, se decidió asignar la cifra total de muertes de los menores de cinco años de edad al grupo de población de menores de un año, puesto que comúnmente se deben a anencefalia o espina bífida, ${ }^{24}$ padecimientos altamente letales. ${ }^{25}$ Con los datos anteriores se calcularon para cada estado $(n=32)$ y para el total de la República Mexicana tasas brutas de mortalidad (por 10000 nacidos vivos), para los 18 años que integran el periodo estudiado (1980-1997). En lo sucesivo nos referiremos a ellas como tasas de mortalidad por DTN. Asimismo, se calculó la mortalidad proporcional para las seis patologías analizadas durante el periodo.

La tendencia de la mortalidad por DTN en el país y en los estados se exploró mediante gráficos suavizados (gráficos no presentados). ${ }^{26}$ En el gráfico nacional y en los gráficos de 21 estados se identificaron dos fases durante el periodo estudiado, con puntos de inflexión en sus tendencias entre 1989 y 1991. En el grupo restante, de 11 estados, se identificó una sola fase en sus tendencias durante todo el periodo 1980-1997.

En ambos grupos, la evaluación de las tendencias de las tasas de mortalidad por DTN, y su cuantificación según el porcentaje de cambio anual (PCA) en la tasa, se realizó mediante un modelo de regresión de Poisson. La dispersión del modelo se valoró mediante el cociente entre el estadístico ji-cuadrado de Pearson y sus grados de libertad, utilizando un enfoque marginal que permitió que la varianza de las observaciones fuera diferente a la media. Además, se analizaron los residuos a partir de la estimación de los residuales de la devianza estandarizados. ${ }^{27}$ La posible presencia de una tendencia se cuantificó a través del coeficiente del PCA, y se valoró su significación estadística gracias a su intervalo de confianza y de la comparación de devianzas mediante la prueba de Fisher.

$\mathrm{Al}$ analizar con el modelo de regresión de Poisson ajustado los estados que presentaron dos fases en la tendencia, se consideró el año 1990 como punto de inflexión para evaluar el PCA en cada una de las fases.

Al hacer la representación gráfica de la mortalidad por DTN, se decidió dividir el periodo de estudio en tres intervalos de seis años (1980-1985, 1986-1991, 1992-1997), elaborando un mapa nacional para cada uno de ellos con la distribución en cuartiles de las tasas de mortalidad correspondientes, con el fin de identificar aquellos estados con las mayores tasas de mortalidad en cada subperiodo.

Finalmente, se calculó la razón entre casos observados y esperados de mortalidad por DTN para cada estado. Los casos esperados se obtuvieron tomando como referencia la tasa nacional estimada con un modelo de regresión de Poisson. Se evaluó el cumplimiento de la hipótesis nula de que el cociente observado/ esperado fuera igual a 1 mediante la prueba de Poisson, que incorpora la sobredispersión estimada en el modelo ajustado para obtener la tasa nacional..$^{28} \mathrm{La}$ razón entre observados y esperados se representó en un mapa con la distribución en cuartiles para el conjunto del periodo estudiado.

El programa utilizado para llevar a cabo el análisis estadístico fue S-Plus $2000 .{ }^{26}$ Para analizar y hacer 
los mapas con información espacial digitalizada se utilizó el programa MapInfo 5.0.29

\section{Resultados}

Durante el periodo 1980-1997, se produjeron en México 21226 muertes por DTN, con un promedio anual de 1179 defunciones (cuadro I). Para el mismo periodo, la tasa anual media de mortalidad a escala nacional fue de 5.8 por 10000 nacidos vivos. La anencefalia fue el tipo de DTN más frecuente $(37.7 \%)$, con una tasa bruta en el periodo estudiado de 2.2 por 10000 nacidos vivos. Seguida por la espina bífida sin hidrocefalia cuya tasa bruta fue de 1.8 por 10000 nacidos vivos, lo que supone $31.6 \%$ de las muertes por DTN. El tercer lugar lo ocupa la espina bífida con hidrocefalia que durante el periodo presentó una tasa bruta de 1.4 por 10000 nacidos vivos y $24.1 \%$ de las defunciones. El encefalocele, el craneorraquisquisis y la iniencefalia fueron menos frecuentes que las anteriores.

Las tasas globales de mortalidad por DTN se incrementan desde el inicio de la serie hasta finales de la década de 1980, a partir de los cuales tienden a descender (figura 1). El punto máximo se alcanza en 1989 con una tasa de 7.8 muertes por 10000 nacidos vivos. $\mathrm{Al}$ analizarlas según el tipo de DTN se observa que en la década de 1980 la anencefalia presenta tasas inferio-

\section{Cuadro I \\ MORTALIDAD INFANTIL* POR DEFECTO Del tubo neural en México, 1980-1997}

$\begin{array}{cccc} & \text { Número } & \text { Tasa bruta } & \text { Mortalidad } \\ \text { de } & \text { por } 10000 & \text { proporcional } \\ \text { Tipo de DTN } & \text { muertes } & \text { nacidos vivos } & (\%)\end{array}$

\begin{tabular}{llll} 
Anencefalia & 7994 & 2.2 & 37.7 \\
\hline Espina bífida sin hidrocefalia & 6707 & 1.8 & 31.6 \\
\hline Espina bífida con hidrocefalia & 5114 & 1.4 & 24.1
\end{tabular}

Encefalocele y otras (iniencefalia

y craneorraquisquisis)

\begin{tabular}{lll}
1411 & 0.4 & 6.6 \\
\hline 21226 & 5.8 & 100
\end{tabular}

* Mortalidad en menores de cinco años de edad emitida por la Secretaría de Salud

res a la espina bífida sin hidrocefalia, pero mantiene un incremento paulatino hasta finales de ese decenio, para situarse como la patología más frecuente durante la década de 1990. Al inicio de dicha década se muestra el valor más alto: 3 muertes por 10000 nacidos vivos, y a partir de entonces se mantiene relativamente constante. Las tasas de mortalidad más altas durante la primera mitad de la serie corresponden a la espina

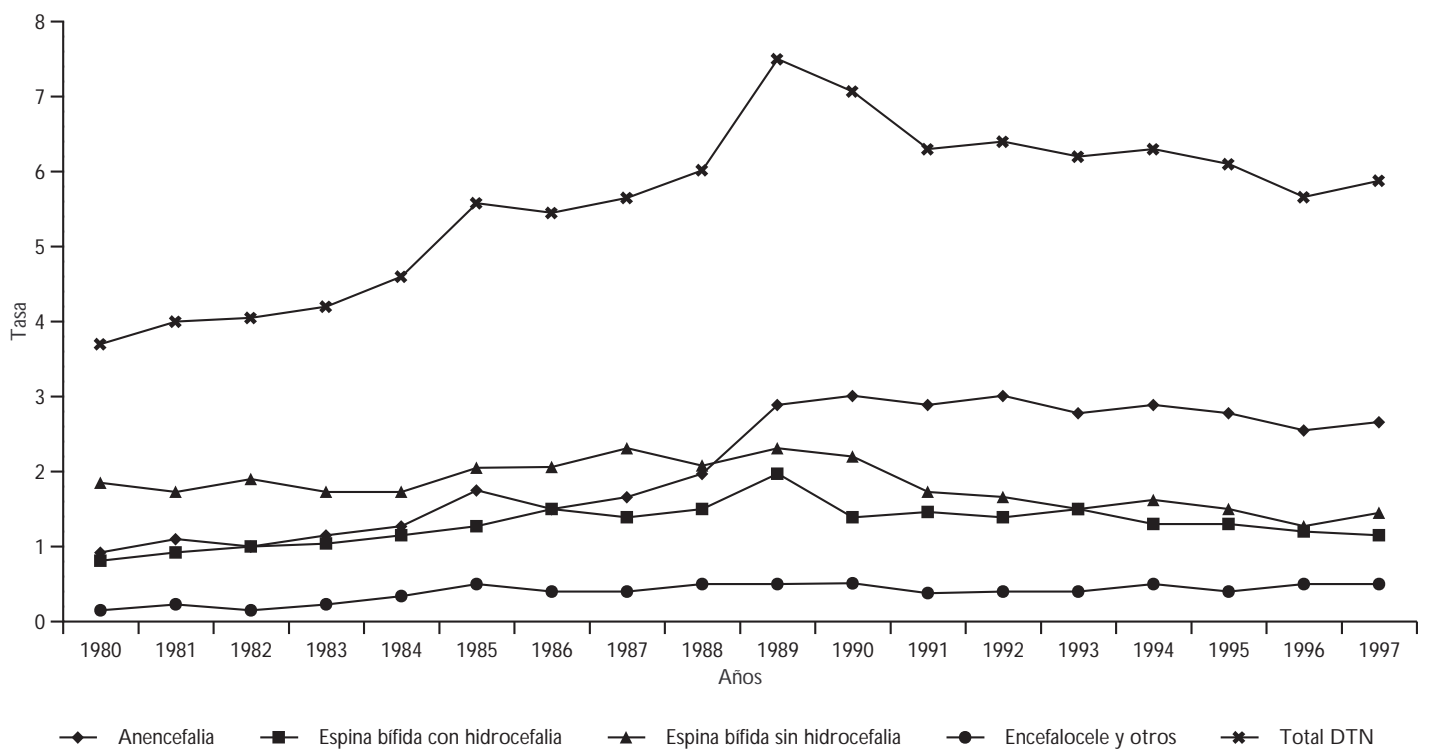

Mortalidad en menores de cinco años de edad DTN : defecto del tubo neural

Figura 1.TASAS de mortalidad infantil* (pOr 10000 nacidos vivos) pOR tipo de defeCto del tUbo neUral en MÉxıco, 1980-1970 
bífida sin hidrocefalia que alcanza su máximo valor en 1987, con una tasa de 2.2 por 10000 nacidos vivos. A partir de 1990 inicia un descenso sostenido hasta el final del periodo, coincidiendo con el incremento de muertes por anencefalia. Situada por debajo de la anencefalia, la espina bífida con hidrocefalia presenta un incremento sostenido en sus tasas de mortalidad desde el inicio de la serie hasta el año 1989, cuando alcanza una tasa máxima de 1.9 por 10000 nacidos vivos; a partir de entonces evoluciona de forma relativamente estable. La cuarta frecuencia corresponde al encefalocele y a craneorraquisquisis e iniencefalia. El primero, durante el periodo estudiado se mantiene constante con tasas de mortalidad de 0.5 por 10000 nacidos vivos o inferiores.

La tendencia nacional de mortalidad por DTN mostró dos fases: una ascendente (1980-1990) y una descendente (1990-1997), ambas estadísticamente significativas (PCA 7.5; IC 95\%:6.5, 8.6 y PCA -2.3; IC 95\%: $-3.6,-0.9$, respectivamente). Este mismo esquema lo presentaron el Distrito Federal y los estados de Hidalgo, Jalisco, Estado de México, Morelos, Nuevo León, San Luis Potosí y Veracruz (cuadro II). Doce estados más (Baja California, Sonora, Chihuahua y Tamaulipas, ubicados en la frontera norte de la República, y Guanajuato, Michoacán, Querétaro, Quintana Roo, Tabasco, Tlaxcala, Yucatán y Zacatecas, ubicados en el centro-sureste) presentaron una primera fase significativamente ascendente y la segunda fase con tendencia estable. Finalmente, el estado de Guerrero, situado al sur de la República Mexicana, mostró una tendencia estable en su primera fase, mientras que la segunda resultó significativamente ascendente.

En el cuadro III se listan los estados que mostraron una sola fase durante todo el periodo; llama la atención la tendencia marcadamente ascendente que muestran los estados de Chiapas, Oaxaca y Puebla, situados en la zona centro-sureste del país, cuyo incremento anual fue de $9.2 \%, 8.7 \%$ y $7.5 \%$, respectivamente. Los estados de Aguascalientes, Baja California Sur, Campeche, Coahuila, Colima, Durango, Nayarit y Sinaloa tuvieron un comportamiento constante (no significativo) durante todo el periodo.

$\mathrm{Al}$ representar geográficamente las tasas de mortalidad por DTN en cada estado (figuras 2A, 2B y 2C) se observó un patrón en el que los estados de Baja California, Guanajuato, Querétaro, Tlaxcala y Yucatán presentaron de forma constante las tasas más elevadas de mortalidad en los tres subperiodos. Asimismo, se identifican estados como Sonora, San Luis Potosí, Oaxaca, Puebla y Quintana Roo, que en alguno de los dos últimos subperiodos (1986-1991 o 1992-1997) se incorporan a las categorías con las tasas más elevadas.

\section{Cuadro II}

Porcentajes de cambio anual con sus intervalos DE CONFIANZA DE 95\%(IC 95\%), DE LAS TASAS DE MORTALIDAD INFANTIL* (POR 10000 NACIDOS VIVOS) POR DEFECTOS DEL TUBO NEURAL EN LOS ESTADOS DE MÉXICO QUE PRESENTARON DOS FASES EN LA TENDENCIA DURANTE LOS PERIODOS 1980-1990 Y 1990-1997

\begin{tabular}{lrrrrrr} 
Estado & PCA 80-90 & \multicolumn{1}{c}{ IC 95\% } & PCA 90-97 & \multicolumn{2}{c}{ IC $95 \%$} \\
Baja California & 12.4 & 8.0 & 17.1 & -0.9 & -5.6 & 4.1 \\
\hline Chihuahua & 7.6 & 3.6 & 11.7 & -3.5 & -8.6 & 1.9 \\
\hline Distrito Federal & 4.2 & 1.3 & 7.2 & -5.5 & -9.7 & -1.0 \\
\hline Guanajuato & 9.4 & 6.1 & 12.8 & -2.7 & -6.7 & 1.3 \\
\hline Guerrero & -0.9 & -5.2 & 3.6 & 14.5 & 8.1 & 21.2 \\
\hline Hidalgo & 10.3 & 6.9 & 13.8 & -5.0 & -9.0 & -0.7 \\
\hline Jalisco & 11.4 & 8.6 & 14.3 & -8.8 & -12.1 & -5.4 \\
\hline Estado de México & 6.1 & 3.1 & 9.2 & -4.8 & -8.4 & -1.0 \\
\hline Michoacán & 4.7 & 2.5 & 7.0 & -2.6 & -5.7 & 0.6 \\
\hline Morelos & 11.4 & 7.4 & 15.6 & -4.9 & -9.4 & -0.2 \\
\hline Nuevo León & 5.7 & 3.6 & 7.8 & -8.7 & -11.6 & -5.7 \\
\hline Querétaro & 6.2 & 2.3 & 10.2 & -0.1 & -4.9 & 5.0 \\
\hline Q uintana Roo & 17.5 & 8.2 & 27.6 & -3.6 & -11.3 & 4.8 \\
\hline San Luis Potosí & 11.9 & 8.4 & 15.4 & -6.7 & -10.5 & -2.6 \\
\hline Sonora & 11.1 & 6.8 & 15.5 & -5.0 & -9.8 & 0.1 \\
\hline Tabasco & 11.7 & 7.3 & 16.2 & -2.2 & -7.0 & 2.8 \\
\hline Tamaulipas & 6.1 & 3.2 & 9.2 & -3.2 & -7.1 & 0.9 \\
\hline Tlaxcala & 9.1 & 5.1 & 13.3 & -0.5 & -5.2 & 4.4 \\
\hline Veracruz & 10.3 & 7.3 & 13.4 & -3.7 & -7.1 & -0.2 \\
\hline Yucatán & 5.9 & 0.6 & 11.6 & -0.7 & -7.4 & 6.6 \\
\hline Zacatecas & 5.3 & 0.2 & 10.6 & 1.4 & -5.3 & 8.7
\end{tabular}

* Mortalidad en menores de cinco años de edad

Valores PCA negativos $=$ tendencia decreciente PCA: porcentaje de cambio anual

Al comparar las muertes observadas en cada estado respecto a la que debiera esperarse según el total nacional para el conjunto del periodo estudiado (figura 2D), se observó que Aguascalientes, Baja California, Colima, Chihuahua, Guanajuato, Jalisco, Estado de México, Morelos, Nuevo León, Puebla, Querétaro, Quintana Roo, Sonora, Tamaulipas, Tlaxcala y Yucatán son los estados cuyas tasas de mortalidad por DTN fueron superiores a la media nacional, pero tan sólo los estados de Guanajuato y Puebla, ubicados en la zona centro del país, presentaron un cociente estadísticamente significativo $(1.59 ; p<0.10$ y $1.58 ; p<0.05$, respectivamente). Las tasas de mortalidad por DTN del resto de los estados fueron inferiores a la media nacional; sin embargo, sólo los estados de Durango y Gue- 
Cuadro III

Porcentajes de Cambio anual con sus intervalos DE CONFIANZA DE 95\%(IC 95\%), DE LAS TASAS DE MORTALIDAD INFANTIL* (POR 10000 NACIDOS VIVOS) POR DEFECTOS DEL TUBO NEURAL EN LOS ESTADOS De México que presentaron una sola tendencia DURANTE EL PERIODO 1980-1997

\begin{tabular}{lccc} 
Estado & PCA 80-97 & \multicolumn{2}{c}{ IC 95\% } \\
A guascalientes & 1.4 & -1.4 & 4.3 \\
\hline Baja California Sur & -2.4 & -6.3 & 1.7 \\
\hline Campeche & 2.3 & -0.8 & 5.4 \\
\hline Coahuila & 3.0 & -1.4 & 7.6 \\
\hline Colima & 1.0 & -2.5 & 4.5 \\
\hline Chiapas & 9.2 & 6.2 & 12.2 \\
\hline Durango & -0.2 & -6.1 & 6.1 \\
\hline N ayarit & 0.5 & -2.5 & 3.7 \\
\hline O axaca & 8.7 & 6.8 & 10.6 \\
\hline Puebla & 7.5 & 5.5 & 9.6 \\
\hline Sinaloa & 0.7 & -2.1 & 3.7
\end{tabular}

* Mortalidad en menores de cinco años de edad

Valores PCA negativos= tendencia decreciente PCA: porcentaje de cambio anual

rrero mostraron significación estadística en la razón observado/esperado $(p<0.10)$.

\section{Discusión}

En el presente estudio, la anencefalia y la espina bífida representaron $93.4 \%$ de los casos de DTN. Mientras que la anencefalia es incompatible con la vida, no contamos con los datos diagnósticos necesarios para determinar la severidad de la espina bífida, ya que sólo fue clasificada con base en su asociación o no con hidrocefalia. Sin embargo, la espina bífida abierta (mielosquisis) es el tipo más común, ${ }^{24}$ y conjuntamente con la espina bífida quística (mielomeningocele) son de pronóstico grave para la vida a corto plazo. 6 Asimismo, en algunos países en desarrollo la mortalidad por espina bífida abierta es cercana a $100 \% .{ }^{25}$ Apoyados en estos antecedentes, y al no contar con los datos específicos de mortalidad infantil por DTN, se aplicó la mortalidad de menores de cinco años de edad al grupo de menores de un año de edad, asumiendo que la sobrestimación que pueda existir en las tasas de mortalidad es mínima.

La anencefalia es el tipo de DTN más frecuente a partir del inicio de la década de 1990, situación que contrasta con el descenso de casos de espina bífida sin hidrocefalia, que pasa a segundo término. Tal circunstancia es difícilmente atribuible a un problema de diagnóstico diferencial dadas las claras diferencias morfológicas entre ellas. Este esquema, que se reproduce en 29 de los 32 estados de México (datos no presentados), se ha observado también en otras latitudes. Así, según datos del registro de malformaciones congénitas de la ciudad española de Barcelona, la anencefalia también fue el tipo de DTN más frecuente durante el periodo 1992-1997, seguida por la espina bífida. ${ }^{30}$ Sin embargo, las altas tasas de mortalidad por anencefalias alcanzadas en México (15.8 por 10000 nacidos vivos) contrastan desfavorablemente con las de otros países de América Latina como Uruguay, Brasil, Chile, Venezuela y Argentina, que oscilan entre 5.6 y 9.5 defunciones por 10000 nacidos vivos. ${ }^{19}$

Al tratarse de un estudio de tendencias, la primera consideración es que el incremento de DTN observado en algunas tendencias pueda no ser real. Así, el incremento podría deberse a una mayor notificación de estas causas de muerte, ya sea por una mejora del sistema de registro o por cambios en los criterios diagnósticos de estas patologías. Aunque tales circunstancias no son descartables, no parece posible que puedan explicar los incrementos descritos, pues se observaron de manera significativa en al menos una fase de la tendencia de 21 estados y durante todo el periodo en tres estados más, todos los cuales tienen un desarrollo sanitario, social y económico desigual. ${ }^{31}$

Por otra parte, no se identificó una circunstancia concreta que explique la inflexión en la tendencia ascendente de aquellos 21 estados. El nivel de conocimiento actual sobre los factores de riesgo asociados con estas patologías durante el periodo estudiado, no permite establecer una hipótesis clara que explique las tendencias ascendentes observadas. Por otro lado, no contamos con datos periódicos sobre la prevalencia de los principales factores de riesgo genéticos y ambientales asociados con los DTN que nos permitan conocer su variabilidad en relación con la mortalidad por estas causas. No obstante, valiéndonos de datos puntuales sobre algunos de ellos, planteamos algunas posibles conjeturas.

La ingestión deficiente de micronutrientes como la metionina, la luteína o el ácido fólico es un factor de riesgo de DTN. ${ }^{10,12,13}$ Los datos de la Encuesta Mexicana de Nutrición de 1999 mostraron que el consumo de folatos estimado en la mujer mexicana entre 12 y 49 años de edad fue inferior a lo recomendado (304 mcg/ día versus $400 \mathrm{mcg} /$ día), con cifras similares, tanto en mujeres embarazadas, como no embarazadas (330 mcg/día y 304 mcg/día, respectivamente) y muy pa- 
2A. Periodo 1980-1985

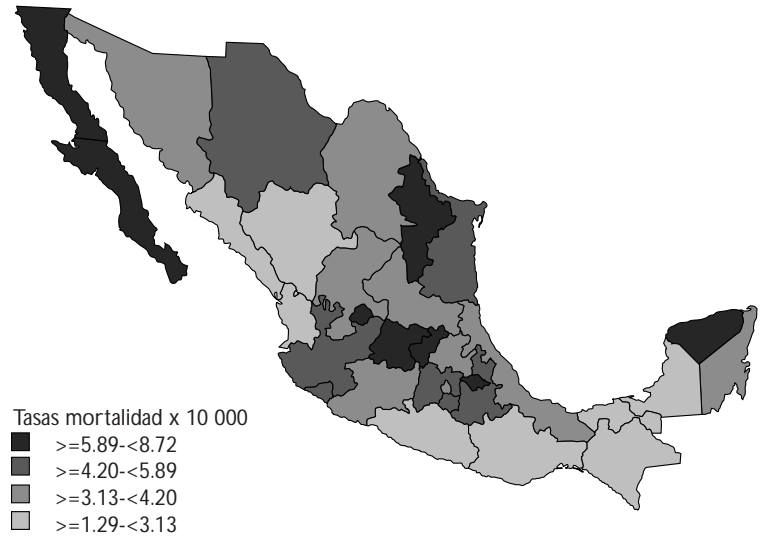

2C. Periodo 1992-1997

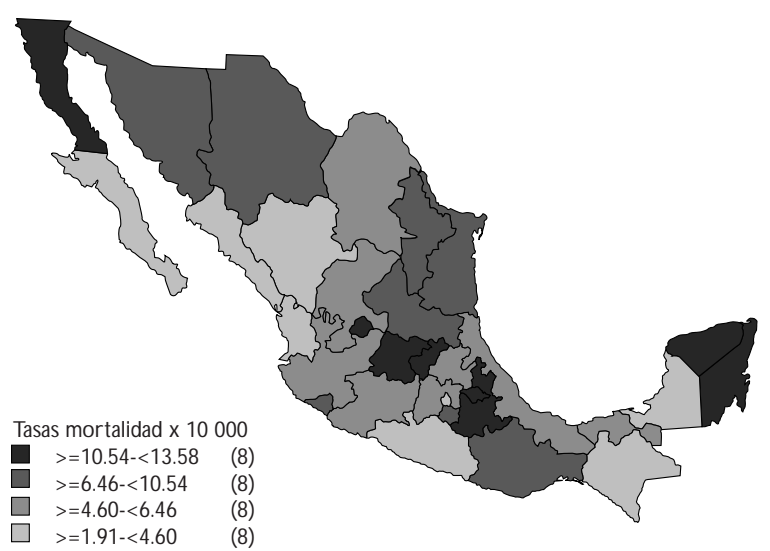

2B. Periodo 1986-1991

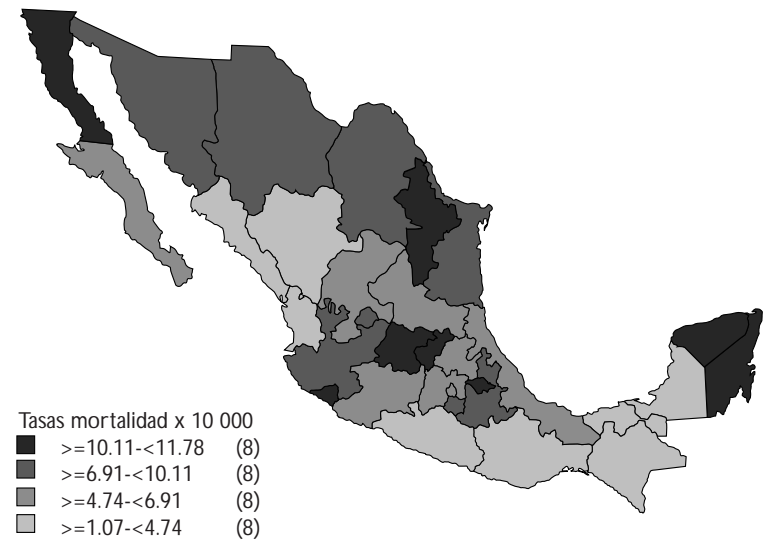

2D. Periodo 1980-1997

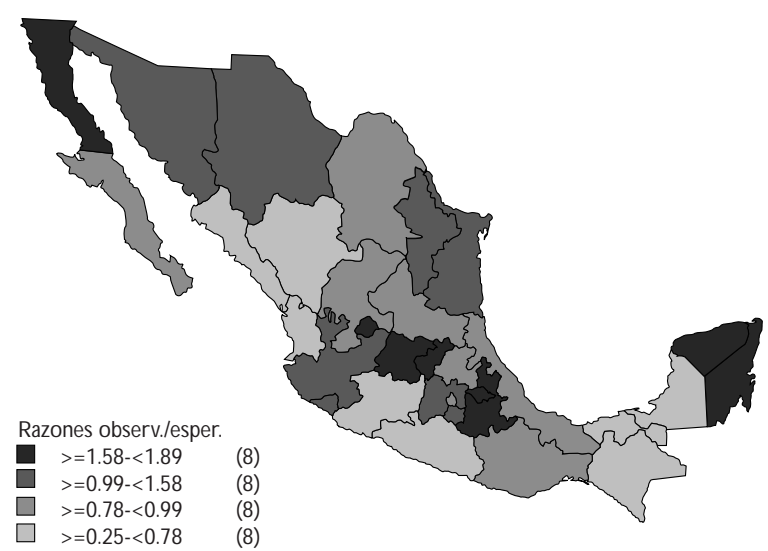

Figura 2.Tasa de mortalidad infantil (por 10000 nacidos vivos) por defectos del tubo neural en México, 1980-1997

recidas a lo largo del territorio nacional. ${ }^{32}$ También se plantea que mutaciones como la $677 \mathrm{CT}$ y la 1298AC, que afectan genes que codifican para folato receptores que intervienen en el metabolismo del ácido fólico, son comunes. ${ }^{33}$ Se estima que en mujeres sanas de diferentes regiones de México la frecuencia de alelo mutado 677 CT es de $59 \%$, siendo más frecuente en las zonas centro y este del país (68\% y $61 \%$, respectivamente). ${ }^{34}$ Sin embargo, otros factores géneticos y ambientales deben estar involucrados, ya que en Italia la mutación $677 \mathrm{CT}$ también es común $(44 \%),{ }^{35}$ pero la tasa de ocurrencia de DTN (anencefalia, espina bífida y encefalocele) es menor que la de México. ${ }^{19}$ Esta situación pudiera deberse en parte a que en Italia y otros países desarrollados la detección in utero de casos de DTN va seguida de un aborto por prescripción médica, dismi- nuyendo así la frecuencia de estos padecimientos en las estadísticas vitales. Se plantea también una posible interacción entre la deficiencia de ácido fólico y polimorfismos genéticos del tipo $677 \mathrm{CT}$, la cual podría estar relacionada con la elevada frecuencia de DTN en México. ${ }^{32}$ Por otra parte, se postula que la obesidad materna es otro factor asociado a DTN. ${ }^{36,37}$ Según datos de esta misma encuesta ${ }^{32} 21 \%$ de las mujeres mexicanas presenta obesidad (índice de masa corporal de 30 o mayor), concentrándose la mayor parte de ellas $(57 \%)$ en la zona centro-sur del país, ${ }^{32}$ zona que asimismo agrupa a los estados con mayores frecuencias de DTN, por lo que éste podría ser otro factor involucrado en los resultados observados.

La exposición ambiental y laboral a compuestos químicos, en particular plaguicidas, es otro factor que 
algunos estudios han asociado a DTN. ${ }^{38-40}$ Aunque en México no existen registros sobre exposición, podemos aproximarnos a la prevalencia de exposición a plaguicidas por medio de la superficie de terreno de cultivo sembrada (hectáreas) en México durante el ciclo 2001$2002,{ }^{41}$ observándose que entre los 11 estados con mayor extensión de superficie sembrada se encuentran los tres con el mayor incremento anual en la tasa de mortalidad por DTN: Chiapas, Oaxaca y Puebla. De hecho, el estado con mayor superficie sembrada es Chiapas, el cual tiene también el mayor PCA (9.2\%) de todo el país. Por el contrario, entre los 11 estados con menor superficie sembrada se encuentran cinco de los ocho (Aguascalientes, Baja California Sur, Campeche, Coahuila, Colima) que no mostraron cambio significativo en las tasas de mortalidad por DTN. La hipótesis de que la actividad agrícola como indicador de exposición a plaguicidas pueda significar un mayor riesgo de engendrar hijos con DTN debiera ser objeto de un estudio específico.

Por otra parte, es importante señalar que los estados de Chiapas, Oaxaca y Puebla, donde el incremento del riesgo de muerte por DTN ha sido mayor, comparten entre sí límites territoriales en la zona centro-sureste. Y que Guerrero, un estado con altos índices de pobreza que comparte límites territoriales con los estados de Puebla y Oaxaca, se une a ellos en una tendencia de mortalidad por DTN significativamente ascendente desde 1990, situación que contrasta con el comportamiento estable que presentó durante los 10 primeros años del estudio.

Por otra parte, en los estados con mayor número de comunidades indígenas marginadas, como es el caso de Chiapas y Oaxaca, donde la accesibilidad a los servicios de salud para estos grupos étnicos es deficiente, pudiera existir un subregistro de casos de DTN. Sin embargo, ambos presentaron durante el periodo de estudio una sola fase ascendente en la tendencia de mortalidad por DTN con un porcentaje de cambio anual positivo (cuadro III), por lo cual, en el caso de que existiese un subregistro constante durante el periodo estudiado, el cambio porcentual anual seguiría siendo el mismo.

Finalmente, al ser la pobreza una condición asociada a los DTN, ${ }^{42}$ todos los factores involucrados deberían analizarse dentro del marco de la pobreza en la que vive cerca de $70 \%$ de la población en México. ${ }^{43}$ Como en muchos otros países, en México también prevalece el esquema de contraste entre los estados del norte y los del sur. Los primeros, con un mayor desarrollo económico, mayor crecimiento industrial, mejor nivel educativo y de vida, mejor nivel de acceso y de atención a la salud, factores que en los estados del sur son mucho menos alentadores. Los estados que presentan una mayor proporción de pobres pertenecen a la zona centro-sur y son aquellos que tienen una elevada concentración de población indígena, entre los que se encuentran Campeche, Chiapas, Guerrero, Hidalgo, Oaxaca, Puebla, Quintana Roo, San Luis Potosí, Veracruz y Yucatán. ${ }^{44}$ Todos ellos, excepto Campeche, Quintana Roo y Yucatán, se ubican también entre los estados con mayor superficie de cultivo sembrada, que muy probablemente es trabajada por campesinos indígenas, hombres y mujeres, y que se encuentran por tanto expuestos a plaguicidas y, del mismo modo, pueden presentar deficiencias de nutrientes y estar expuestos a otros potenciales factores de riesgo.

Actualmente, con la finalidad de disminuir la frecuencia de estos defectos se promueven diversas medidas de prevención primaria: desde la educación para la salud, la orientación nutricional y el consejo genético, hasta la suplementación con ácido fólico. ${ }^{45}$

No obstante, es necesario el planteamiento de hipótesis que originen líneas de investigación específicas que permitan evaluar los diversos factores de riesgo involucrados.

\section{Referencias}

1. 0 rganización Panamericana de la Salud. 0 rganización Mundial de la Salud. La salud en las A méricas.W ashington, D C: PA HO -W HO , 1998. Publicación Científica N 0.569 (I).

2. Pan American Health O rganization. El Paso Field O ffice. US-Mexico border. En línea. URL disponible en: http://www.fep.paho.org/epibul5/ p2.htm. Fecha de acceso 7 de febrero de 2002.

3. Edmonds LD, Layde PM, James LM, Flynt JW, Erickson JD, 0 zkley G P. Congenital malformations surveillance:Two american systems. Int J Epidemiol 1981;10(3):247-252.

4. Murray CJL, López AD. Mortality by cause for eight regions of the world: G lobal burden of disease study. Lancet 1997; 349:1269-1276. 5. Secretaría de Salud, Dirección General de Epidemiología e Informática, Instituto N acional de Estadística, Geografía e Informática. Estadísticas vitales. Capítulo Mortalidad. México, DF: SSA, 1998. 6. Moore KL, Persaud TVN . Embriología clínica 5a ed. México, D F: N ueva Editorial Interamericana- McG raw-Hill, 1995:154.

7. López SA.Atención a la salud en México (editorial). Prevención de los defectos del nacimiento. Foro Silanes 2001;(11):2.

8. Sadler TW. Embriología médica. 7ạ ed. México, DF: Editorial Médica Panamericana, 1997:114.

9. Brent RL, Beckman DA. Environmental teratogens. Bull NY Acad Med 1990;66:123-163.

10. Garrow JS, James W PT, Ralph A. Human nutrition and dietetics. $10^{\text {th }}$ ed. Londres: Churchill-Livingstone, 2000:274.

11. Shaw GM,Todoroff K, Schaffer DM, Selvin S. Periconceptional nutrient intake and risk for neural tube defect-affected pregnancies. Epidemiology 1999;10(6):711-716.

12. Barber RC, Lammer EJ, Shaw GM, Greer KA, Finnell RH. Minireview. The role of folate transport and metabolism in neural tube defect risk. Mol Genet Metab 1999;66:1-9. 
13. Finnell RH, Greer KA, Barber RC, Piedrahita JA, Shaw GM, Lammer EJ. N eural tube and craniofacial defects with special emphasis on folate pathway genes. Crit Rev 0 ral Biol Med 1998;9(1):38-53.

14. García AM. 0 ccupational exposure to pesticides and congenital malformations: A review of mechanisms, methods, and results. Am J Ind Med 1998:33:232-240.

15. Schardein JL. Pesticides. En: Schardein JL, ed. Chemically induced birth defects. $2^{\text {nd }}$ Ed. N ueva York (N Y): Marcel D ecker, 1993:675-721. 16.W orld Health 0 rganization. Public health impact of pesticides used in agriculture. Ginebra:W HO 1990.

17. Rowland AS. Pesticides and birth defects. Epidemiology 1995;6(1): 6-7.

18. W orld Health 0 rganization. Principles for evaluating health risks to progeny associated with exposure to chemicals during pregnancy. Environmental Health Criteria. Ginebra:W HO 1984 19. O rganización Mundial de la Salud.W orld Atlas of Birth D efects. En línea. URL disponible en: http://www.who.int/home-page/index.es.shtml. Fecha de acceso 2 de mayo de 2002.

20. Martínez-Villarreal LE,A yala-Alvarado C, Limón-Benavides C. D efectos congénitos y mortalidad en el estado de $\mathrm{N}$ uevo León. Medicina Universitaria 1990;1:113-117.

21. Busam KJ, Roberts DJ, Golden JA. C linical teratology counselling and consultation case report:Two distinct anterior neural tube defects in a human fetus. Evidence for an intermittent pattern of neural tube closure. Teratology 1993;48:399-403.

22. Tapia-C onyer R. Importancia de los defectos al nacimiento en México. Foro Silanes 2001;(11):3.

23. 0 rganización Panamericana de la Salud. O rganización Mundial de la Salud. C lasificación Internacional de Enfermedades. Manual de la clasificación estadística internacional de enfermedades, traumatismos y causas de defunción. N ovena revisión 1975.W ashington, DC: PAHO W HO , 1978; Publicación Científica N 0. 353:443.

24. 0 'Rahilly R, Muller F. Embriología y teratología humanas. Barcelona, España: Masson, 1998:430.

25. Moore CA, Li S, Li Z, Hong S, Gu H, Berry Rj et al. Elevated rates of severe neural tube defects in a high-prevalence area in $\mathrm{N}$ orthern $\mathrm{C}$ hina. Am J Med Genet 1997;73:113-118.

26. S-Plus 2000 Guide to Statistics,Volume 1, D ata A nalysis Products Division, Seattle (W A): MathSoft, 1999.

27. Chambers JM, H astie TJ, Ed. Statistical model in S.W asdworth and Brooks Cole Advanced Books and Software. Pacific Grove:AT\& Bell Laboratories, 1992.

28. Benach J,Yasui Y, Borrell C, Rosa E, Pasarín MI, Benach N et al. Atlas de mortalidad en áreas pequeñas de España (1987-1995). Anexo II. Métodos estadísticos. Barcelona: Universitat Pompeu Fabra/MSD, 2001. 29. MapInfo Professional User's Guide.Troy (N Y): MapInfo Corporation, 1995. Disponible en: http://www.columbia.edu/acis/eds/gis/mapinfo65pdf/ MIUG.pdf

30. Salvador J, Llandonosa A, C unillé M, C abré A, Ricart M, Alonso S et al. Registro de defectos congénitos de la ciudad de Barcelona. Informe anual 1999. Barcelona: Instituto Municipal de Salud Pública,

Ayuntamiento de Barcelona, 1999.

31. Secretaría de Salud de México. Estadísticas en salud. Recursos.

Indicadores de recursos. En línea. URL disponible en: $h$ ttp://

www.ssa.gob.mx. Fecha de acceso 8 de marzo de 2002.
32. Secretaría de Salud. Instituto N acional de Salud Pública de México. Encuesta N acional de Salud 1999. En línea. URL disponible en: http:// www.insp.mx. Fecha de acceso 8 de marzo de 2002.

33. Botto LD, Moore CA, Khoury MJ, Erickson JD. Review articles. Medical progress. N eural tube defects. N Engl J Med 1999;341(20): 1509-1519.

34. Mutchinick O M, López MA, Luna L, W axman J, Babinsky VE.High prevalence of the thermolabile methylenetetrahydrofolate reductase variant in Mexico:A country with a very high prevalence of neural tube defects. Mol Genet Metab 1999:68:461-467.

35. Botto LD, Yang Q . 5, 10-Methylene tetrahydrofolate reductase (MTHFR) gene variants and congenital anomalies:A huge review. Centers for Disease Control and Prevention. $N$ ational $C$ enter for Environmental Health. Division of Birth D efects and Pediatric Genetics. Atlanta (GA), 1999. En línea. URL disponible en: http://www.cdc.gov/ genomics/hugenet/reviews.htm. Fecha de acceso 2 de mayo de 2002. 36. Shaw GM, Velie EM, Schaffer DM. Risk of neural tube defect-affected pregnancies among obese women. JAMA 1996;275(14):1093-1096. 37.W aller DK, Mills JL, Simpson JL. A re obese women at higher risk for producing malformed offspring? Am J 0 bstet Gynecol 1994;170: 541-548.

38. Shaw GM,W asserman CR, 0 'Malley CD, N elson V, Jackson RJ. Maternal pesticide exposure from multiple sources and selected congenital anomalies. Epidemiology 1999;10(1):60-66.

39. Kristensen P, Irgens LM, Andersen A, Snellingen BA, Sundheim L. Birth defects among offspring of N orwegian farmers 1967-1991. Epidemiology 1997;8(5):537-544

40. García AM, Fletcher T, Benavides FG , 0 rts E. Parental agricultural work and selected congenital malformations. Am J Epidemiol 1999; 149(1):64-73.

41. Secretaría de Agricultura, Ganadería, Desarrollo Rural, Pesca y Alimentación. Servicio de Información y Estadística Agroalimentaria y Pesquera.Avance de siembras y cosechas 2001-2002 México. URL disponible en: http://www.siea.sagarpa.gob.mx/indexgiras2.html. En línea. Fecha de acceso 8 de marzo de 2002.

42.W asserman CR, Shaw GM, Selvin S, Gould JB, Syme SL. Socioeconomic status, neighborhood social conditions, and neural tube defects. Am J Public Health 1998;88:1674-1680.

43. 0 rganización Panamericana de la Salud. O rganización Mundial de la Salud. Perfil de salud para México. En línea. URL disponible en: http:// 165.168.1.110/spanish/sha/prflmex.html. Fecha de acceso 8 de marzo de 2002.

44. Centro de Investigación y Estudios Superiores en Antropología Social. Banco Interamericano de Desarrollo. Perfil de los pueblos indígenas de México: ubicación. En línea. URL disponible en: http:// www.ciesas.edu.mx/biddf/ini/nacional/02 ubicacion.html. Fecha de acceso 8 de marzo de 2002

45. Barber R, Hendricks K, Joggerst B, Larsen R, Suárez L, Finnel R. Investigation of folate pathway gene polymorphisms and the incidence of neural tube defects in aTexas Hispanic population. Mol Genet Metab 2000; 70(1):45-52. 